\title{
Zur Verwendung einiger Transportverben im Deutschen und im brasilianischen Portugiesisch
}

HARDARIK BLÜHDORN

\section{Abstract}

This article summarizes results of an empirical study on the use of so called verbs of transportation in German and Brazilian Portuguese. Such verbs constantly cause difficulties and mistakes in the language production of non-native speakers. The paper presents four observations on the grammar (verb prefixes, prepositions), semantics (places and paths) and pragmatics (deixis) of verbs of transportation in the two languages. It leads to the conclusion that Brazilian learners tend to have more difficulties with the morphology and syntax of German transportation verbs, whereas German learners tend to have more difficulties with the pragmatics of the corresponding verbs in Brazilian Portuguese. Difficulties with the specification of places and paths can be observed in both directions, but they lead to unidiomatic usage rather than to outright mistakes.

\section{Einleitung}

Der vorliegende Aufsatz befasst sich mit einer Gruppe von Verben im Deutschen und im brasilianischen Portugiesisch, die ich informell als Transportverben bezeichne. Im Portugiesischen handelt es sich um Verben wie apanhar, buscar, pegar, tomar, levar und trazer, im Deutschen um deren Entsprechungen wie holen, abholen, bringen, mitbringen, nehmen und mitnehmen.

Die portugiesischen Transportverben sind für deutsche Lerner ebenso wie die deutschen Transportverben für brasilianische Lerner eine Quelle persistenter Fehler, wobei unter Fehlern zum einen klassischer ungrammatischer Gebrauch, zum anderen unidiomatischer, also für Muttersprachlerohren seltsamer Gebrauch verstanden werden soll.

Der Aufsatz präsentiert Ergebnisse einer Untersuchung, die am Institut für Deutsch der Universidade de São Paulo, Brasilien, durchgeführt wurde. ${ }^{1}$ Es ging darum, den Gebrauch der deutschen und portugiesischen Transportverben in der Muttersprache und in der Fremdsprache unter grammatischem, se- 
mantischem und (teilweise) pragmatischem Blickwinkel zu beschreiben und zu vergleichen. Dabei sollten vor allem die typischen Fehler untersucht und erklärt werden, die Sprecher der jeweils anderen Sprache mit diesen Verben produzieren. Als weiteres Ziel sollten Vorschläge für die Fremdsprachendidaktik abgeleitet werden. Hierauf kann im vorliegenden Rahmen aber nicht eingegangen werden.

Für die Untersuchung wurde ein Korpus von ca. 2.500 Sätzen mit insgesamt dreißig deutschen und dreißig portugiesischen Verben erhoben. Als Informanten dienten deutsche Portugiesisch-Studenten der Universität ErlangenNürnberg, brasilianische Deutsch-Studenten der Universidade de São Paulo und brasilianische Teilnehmer der Mittel- und Oberstufen-Deutschkurse des Goethe-Institutes São Paulo. Erhoben wurden bei den deutschen und den brasilianischen Informanten jeweils drei Typen von Daten: Eine Gruppe von Informanten wurde gebeten, je zwei typische Sätze mit vorgegebenen Transportverben der Muttersprache zu bilden (als Mittel, um deren idiomatischen Gebrauch festzustellen); eine andere Gruppe sollte je zwei Sätze mit vorgegebenen Transportverben der Fremdsprache produzieren (um deren Gebrauch in grammatischen Konstruktionen zu dokumentieren); eine dritte Gruppe sollte vorgegebene Sätze mit den betreffenden Verben aus der Muttersprache in die Fremdsprache übersetzen (um die Selektion der Verben in der Fremdsprache zu dokumentieren). ${ }^{2}$ Als ergänzende Informationsquelle zum idiomatischen Gebrauch der Verben wurden die einschlägigen Artikel aus Wörterbüchern, insbesondere Valenzwörterbüchern, beider Sprachen (Buarque de Holanda Ferreira 1986, Borba et al. 1991, Fernandes 1991, Luft 1995; Helbig/Schenkel 1973, Engel/Schumacher 1978, Gerling/Orthen 1979, Schumacher 1986, Duden 1989, Schröder 1992, Götz et al. 1993) sowie aus zweisprachigen Wörterbüchern (Schau 1985, Irmen/Beau 1986, Klare 1988, Keller 1994) herangezogen.

Im folgenden werden aus den Ergebnissen der Untersuchung vier Beobachtungen herausgegriffen, die die Bereiche der Morphologie und Syntax, der Semantik und der Pragmatik berühren. Ich werde mich dabei weitgehend auf die eingangs genannten zwölf Verben beschränken, die einen kleinen, aber zentralen Ausschnitt aus dem Objektbereich der Gesamtuntersuchung bilden.

\section{Vier ausgewählte Beobachtungen}

\subsection{Präfixverben und Simplizia}

Die portugiesischen Verben apanhar, buscar, pegar, tomar, levar und trazer sind, gegenwartssprachlich betrachtet, Simplizia. Unter ihren deutschen Entsprechungen holen, abholen, bringen, mitbringen, nehmen und mitnehmen sind drei Simplizia und drei Präfixbildungen. ${ }^{3}$ Im Deutschen kann eine große Zahl weiterer Transportverben durch Präfigierung von denselben Simplizia abgelei- 
tet werden (z. B.: herholen, hinbringen, wegnehmen usw.). Die entsprechenden Möglichkeiten im Portugiesischen sind sehr beschränkt.

Das Deutsche ist, anders als das Portugiesische, außerordentlich reich an Präfixverben (vgl. Schröder 1992: 9 f.). Die deutschen Verbpräfixe haben unterschiedliche Funktionen. Manche Präfigierungen sind gegenwartssprachlich kaum noch mit den dazugehörigen Simplizia bedeutungsverwandt (z. B. überlegen und legen). In anderen Fällen ändert sich lediglich der Aspekt, während die Bedeutung im großen und ganzen gleich bleibt (z. B. erlernen und lernen). Meistens haben Präfixverben einen beschränkteren Verwendungsbereich als Simplizia, z. B. beschränkt auf bestimmte Objektklassen (wie bei $z u$ bereiten gegenüber bereiten) oder bestimmte Vorgangstypen (wie bei abrechnen gegenüber rechnen). Oft unterscheiden Präfixverben und Simplizia sich in der Valenz, wobei manche Präfixverben mehr Leerstellen eröffnen als die entsprechenden Simplizia (z. B. verarbeiten gegenüber arbeiten), manche weniger (z. B. einkaufen gegenüber kaufen), manche einfach andere (z. B. beantworten gegenuiber antworten) (vgl. insgesamt: Eichinger 1989; Fleischer/Barz 1992: 289 ff.; Duden 1998: 451 ff.).

Durch ihren beschränkteren Verwendungsbereich sind Präfixverben im Deutschen gewöhnlich konkreter als Simplexverben. Dem Muttersprachler erlauben sie es, das Gemeinte mit sparsamen Mitteln präzise auszudrücken. Deshalb werden Präfixverben häufig gegenüber Simplizia bevorzugt. Mehrere unserer deutschen Informanten haben auf die Aufforderung, typische Sätze mit den Simplexverben holen, nehmen und bringen zu bilden, ohne es zu bemerken, Sätze mit den Präfixverben abholen, mitnehmen und mitbringen gebildet. Dagegen hat kein einziger deutscher Informant versehentlich statt eines geforderten Präfixverbs ein Simplexverb verwendet.

Im Portugiesischen ist die Situation anders. Zum einen bietet es wesentlich weniger Präfigierungsmöglichkeiten und besitzt somit weitaus weniger Präfixverben. Als Transportverben kommen zum Beispiel retirar, recolher, remover, deslocar und transferir in Betracht. Solche Verben haben im brasilianischen Portugiesisch einen eher formellen bzw. technischen Charakter. Unsere brasilianischen Informanten haben sie in Akzeptabilitätstests verschiedentlich zurückgewiesen und ihre Ersetzung durch Simplexverben wie tirar, colher, levar etc. vorgeschlagen.

Die Vorliebe der brasilianischen Informanten für Simplexverben blieb auch in ihrer Produktion im Deutschen als Fremdsprache erhalten. Zum einen verwendeten sie im Satzbildungsexperiment manchmal unbemerkt Simplizia statt geforderter Präfixverben (z.B. holen statt abholen) und verhielten sich damit genau entgegengesetzt zu den deutschen Informanten. In keinem einzigen Fall wurde von ihnen versehentlich ein Präfixverb statt eines Simplexverbs verwendet. Zum anderen benutzten sie im Übersetzungsexperiment häufig unidiomatisch Simplexverben statt idiomatischer Präfixverben, z. B.: 
Von deutschen Lernern ist umgekehrt zu erwarten, dass sie im Portugiesischen $\mathrm{zu}$ übertriebenem Gebrauch von Präfixverben, also von formellen oder technischen Verben neigen - allerdings erst, wenn sie die betreffenden Verben gelernt haben, also relativ spät, da diese ja nicht zum portugiesischen Anfängerwortschatz zählen. Wenn derartige Fehler auftreten, stehen dem Lerner somit wahrscheinlich schon die gängigeren Simplizia zur Verfügung und damit die Mittel, um seine Fehler selbst zu korrigieren.

Die erste Beobachtung lautet somit: Der Gebrauch von Simplizia anstelle von präfigierten Verben ist eine typische Fehlerquelle für deutschlernende Brasilianer. Neben dem Motiv, dass im Portugiesischen Simplizia bevorzugt werden (Interferenz; in Bezug auf die Zielsprache Deutsch ein externes Motiv), gibt es für deutschlernende Brasilianer drei zielsprachinterne Motive, Präfixverben zu meiden:

1. Bekanntlich sind im Deutschen einige Präfixverben trennbar, andere nicht. Trennbarkeit von Verben ist im Portugiesischen nicht vorgesehen, und die diesbezüglichen Regeln des Deutschen sind relativ kompliziert.

2. Abgetrennte Verbpräfixe müssen meistens weit von der Verbbasis entfernt am Satzende serialisiert werden, was die Durchschaubarkeit der betreffenden Strukturen vermindert.

3. Die Regeln der Verb-Präfigierung sind einerseits erkennbar produktiv, andererseits chaotisch (polyanalog und polyfunktional) und dadurch abschreckend.

Die Gesamtheit dieser Motive führt dazu, dass brasilianische Deutschlerner Simplexverben bevorzugen. Häufig ergeben sich daraus Fehler (wie in Beispiel (1)) oder unidiomatische Formulierungen.

\subsection{Präpositionen}

Transportverben dienen zur sprachlichen Darstellung von Transportsachverhalten. Wir wollen annehmen, dass Transportsachverhalte generell so funktionieren, dass ein Gegenstand $x$ (Patiens) zunächst an einem Ort A situiert ist, dann von diesem entfernt wird und als Ergebnis des Transportes an einem Ort B situiert ist. Es kann sich somit, je nach Betrachtungsweise, der Bedarf ergeben, verschiedene Typen von Orten sprachlich darzustellen. Wenn man den Weg bzw. die Bewegung im Auge hat, so kann man Ausgangsorte, von denen der Gegenstand wegbewegt wird, und Zielorte, zu denen er hinbewegt wird, unterscheiden. Hat man den Gegenstand selbst im Auge, so kann man beide Orte auch als Situierungsorte auffassen, an denen sich der Gegenstand zu verschiedenen Zeitpunkten befindet (vgl. dazu auch Blühdorn 1993a: $154 \mathrm{ff}$.). 
Ein wichtiges Sprachmittel zur Darstellung von Orten sind im Portugiesischen wie im Deutschen präpositionale Ausdrücke. Im Portugiesischen finden wir eine relativ einfache Verteilung der Präpositionen auf die drei Typen von Orten. Situierungsorte werden sehr häufig mit $\mathrm{em}$ angezeigt, wie in (2a), Ausgangsorte mit $d e$, wie in (2b) und Zielorte mit $a$ bzw. pra (para), wie in (2c):
a. $\quad n a^{4}$ escola, na praia, na casa do Pedro, em São Paulo, em casa
b. da escola, da praia, da casa do Pedro, de São Paulo, de casa, daqui
c. à escola, à praia, pra casa do Pedro, pra São Paulo, pra casa, pra lá

Im Deutschen sind die Verhältnisse demgegenüber viel komplizierter (vgl. etwa Helbig/Buscha 1986: 412ff.; Duden 1998: $386 \mathrm{ff}$.). Situierungsorte werden, abhängig von sachlichen und sprachlichen Faktoren, mit sehr unterschiedlichen Präpositionen angezeigt (3a), ebenso Ausgangsorte (3b) und Zielorte (3c):
a. in der Schule, am Strand, bei Peter, in São Paulo, zu Hause
b. aus der Schule, vom Strand, von Peter, aus oder von São Paulo, von zu Hause, von hier
c. in die Schule, an den Strand, zu Peter, nach São Paulo, nach Hause, nach dort

Hinzu kommt, dass die deutschen Präpositionen unterschiedliche, teilweise wechselnde Kasus fordern und dass die meisten von ihnen polyfunktional sind. So werden in und an (und die übrigen sogenannten Wechselpräpositionen, die hier einfachheitshalber ausgespart bleiben sollen) zum Anzeigen von Situierungsorten mit dem Dativ, zum Anzeigen von Zielorten mit dem Akkusativ konstruiert. Immerhin werden diese Präpositionen in beiden Fällen mit denselben Substantivgruppen kombiniert. Die Präposition $z u$ dagegen wird zur Anzeige von Situierungsorten und zur Anzeige von Zielorten mit verschiedenen Substantivgruppen kombiniert (einmal mit der Bezeichnung für die eigene Wohnung, das andere Mal mit Bezeichnungen für Personen, Institutionen, Berge, Flüsse etc.). Sie fordert dafür in beiden Fällen den Dativ. Zum Anzeigen von Ausgangsorten verwendet man die Präposition von mit Elementen, die bei der Anzeige von Situierungsorten mit an, bei, zu oder ohne Präposition konstruiert werden, und die Präposition aus bei Elementen, die bei der Anzeige von Situierungsorten mit in oder $z u$ konstruiert werden. Tabelle 1 gibt einen schematischen Überblick.

Wiederum sind es die Brasilianer, für die sich in diesem Bereich Schwierigkeiten ergeben. Im allgemeinen versuchen sie, den portugiesischen Präpositionen $e m$, de und a/pra je eine deutsche Präposition zuzuordnen und diese 
Tabelle 1. Präpositionen zur Kennzeichnung von Orten im Portugiesischen und im Deutschen

\begin{tabular}{llll}
\hline Ausgangsort & Situierungsort & Zielort & \\
\hline$d e$ & $e m$ & $a$ & escola \\
de & $e m$ & $a$ & praia \\
$d e$ & $e m$ & pra & casa do Pedro \\
$d e$ & $e m$ & pra & São Paulo \\
de & $e m$ & pra & casa \\
de & - & pra & lă \\
aus & in DAT & in + AKK & Schule \\
von & $a n+$ DAT & an + AKK & Strand \\
von & bei & $z u$ & Peter \\
aus $/$ von & in & nach & São Paulo \\
von zu & $z u$ & nach & Hause \\
von & - & nach & dort \\
\hline
\end{tabular}

kontextunabhängig als Äquivalent einzusetzen. Meist wählen sie für de die Präposition von, für em die Präposition in und für a/pra die Präposition für (die im deutschen Ortsanzeigesystem überhaupt nicht vorkommt) oder auch $z u$. Die Variante von - in - für hat den Vorteil, dass sie sich partiell (bei Objekten und Genitivattributen) auch zur Substitution des deutschen Kasussystems verwenden lässt (von + DAT für den Genitiv, für + AKK für den Dativ); die Variante von - in - zu hat den Vorteil, dass alle drei Präpositionen den Dativ fordern, also die Schwierigkeiten mit der Rektion wegfallen. Typische Fehler brasilianischer Deutschlerner aus unserem Korpus sind:

(4) Sie hat diese Rose von unserem Garten gepflückt. (statt: ... in unserem Garten gepflückt oder ... aus unserem Garten geholt)

(5) Bitte, holen Sie einen Stuhl von dem anderen Raum. (statt: ... aus dem anderen Raum)

(6) Die Mutter kann nicht im Eigentumswohnungstreffen bleiben (statt: ... auf dem Treffen), denn sie muß ihren Sohn in der Schule bringen. (statt: ... in die Schule)

(7) Wir haben nur einen leichten Koffer in der Reise genommen. (statt: ... auf die Reise mitgenommen)

(8) Sie bringen das Essen für Abschlußfest. (statt: ... zum Abschlußfest mit)

(9) Für die deutsche Stunde bringe ich immer ,Sprachbrücke“. (statt: In den Deutschunterricht bringe ich ... mit.) 
(10) Die Gefangenen wurden zu einem Gefängnis auf dem Land versetzt. (statt: ... in ein Gefängnis ... verlegt)

(11) Der Atommüll ist zur Angra geschleppt. (statt: ... nach Angra gebracht worden)

Die zweite Beobachtung lautet entsprechend: Präpositionen und Kasus bei Orts- und Wegangaben sind bei den Transportverben eine weitere typische Fehlerquelle für deutschlernende Brasilianer. Hier ist das zielsprachexterne Motiv (Interferenz mit dem einfacheren Präpositionensystem des Portugiesischen) die Kehrseite des zielsprachinternen Motivs (komplizierte Struktur des deutschen Präpositionensystem, das zudem mit der als schwierig bekannten und im Portugiesischen fehlenden Deklination zusammenhängt).

\subsection{Situierungsort, Ausgangsort und Zielort}

Auf den zweiten Blick ist das Ortskennzeichnungssystem des Portugiesischen doch nicht ganz so einfach, wie es zunächst scheint. So finden sich in unserem Korpus nebeneinander apanhar as frutas do pomar [das Obst aus dem Obstgarten holen] und apanhar algumas frutas no pomar [einige Früchte im Obstgarten holen/pflücken]. Offenbar wird hier der Obstgarten einmal als Ausgangsort des bewegten Gegenstandes, das andere Mal als Situierungsort der Transporthandlung betrachtet.

Auch der Ort, an dem der Gegenstand sich am Ende des Transportes befindet, kann gelegentlich außer mit a/para auch mit $e m$ angezeigt werden. So findet sich im Korpus (12a) neben (12b):
a. Márcia levou um amigo para casa.
'Márcia hat einen Freund mit nach Hause genommen. oder ... nach Hause gebracht.'
b. Carlos foi levá-los em casa.
'Carlos ist gefahren, um sie nach Hause zu bringen.'

Eine Hypothese, um diese Datenlage zu erklären, könnte sein, dass im brasilianischen Portugiesisch die Unterscheidung zwischen Situierungsort und Wegort weniger wichtig genommen wird als im Deutschen. Dafür spricht, dass brasilianische Sprecher auch die Frageadverbien onde [wo] und aonde [wohin] häufig vertauschen. ${ }^{5}$

Gegen die alleinige Erklärungskraft dieser Vermutung spricht allerdings das Verb buscar, das eine Kennzeichnung des Ausgangsortes (wenn man den Wörterbüchern und unseren Informanten trauen darf) nur mit em und nicht mit de erlaubt:

Vou buscar meu filho na escola. (*da escola)

'Ich werde meinen Sohn in der Schule abholen.' 
Sein deutsches Äquivalent abholen zeigt dagegen die gleiche Alternation wie apanhar:

a. Die Mutter holt das Kind in der Schule ab.

b. Die Mutter holt das Kind von/aus der Schule ab.

Andere deutsche Verben, z. B. abliefern oder anbringen, verhalten sich ähnlich wie buscar, allerdings auf den Zielort bezogen: Sie erlauben die Kennzeichnung mit Elementen, nach denen man wo fragt, aber nicht die Kennzeichnung mit Elementen, nach denen man wohin fragt:

Die Waren sind im Erdgeschoss (*ins Erdgeschoss) abzuliefern.

Bringen Sie den Zettel doch am Schwarzen Brett (?ans Schwarze Brett) an.

Offenbar gibt es im Portugiesischen wie im Deutschen die Möglichkeit, das Zusammenspiel zwischen Transport und Situierung perspektivisch zu differenzieren. In (14a) wird der Ort hervorgehoben, an dem die Mutter das Kind antrifft, in (14b) die Bewegung des Kindes von diesem Ort weg. Entsprechend wird in (12a) die Bewegung betont und in (12b) der Ort, an dem sie zum Stillstand kommt. Allerdings sind die Differenzierungsmöglichkeiten, wie die Beispiele buscar, abliefern und anbringen zeigen, unter Umständen verbspezifisch eingeschränkt.

Wir können, um die Verhältnisse weiter aufzuklären, die oben eingeführte konzeptuelle Unterscheidung zwischen Ausgangs-, Ziel- und Situierungsorten, nun durch die grammatische Unterscheidung zwischen wo-, woher- und wohinBestimmungen ergänzen. Unter einer wo-Bestimmung ist eine Bestimmung zu verstehen, die mit wo bzw. onde erfragt wird, also etwa in der Schule oder na escola; entsprechend werden woher- und wohin-Bestimmungen mit woher/de onde und wohin/aonde erfragt.

Wie wir gesehen haben, können Ausgangsorte im Portugiesischen wie im Deutschen durch woher-oder durch wo-Bestimmungen und Zielorte durch wohin- oder wo-Bestimmungen spezifiziert werden. Die Spezifizierung durch eine wo-Bestimmung lässt dabei jeweils den Wegort als Situierungsort erscheinen. Eine Spezifizierung von Ausgangsorten durch wohin-Bestimmungen oder von Zielorten durch woher-Bestimmungen ist in beiden Sprachen ausgeschlossen.

Es ist nun interessant, für die hier untersuchten sechs portugiesischen und sechs deutschen Verben die in den Wörterbüchern und in den Daten unseres Korpus vorkommenden Möglichkeiten der Ortsspezifizierung zusammenzustellen (Tabelle 2).

Quantitative und qualitative Indizien deuten darauf hin, dass es zu jedem der untersuchten Verben eine Frage bezüglich des Transportweges bzw. der 
Tabelle 2. Möglichkeiten der Bestimmung von Wegorten bei portugiesischen und deutschen Transportverben

\begin{tabular}{lll}
\hline & Ausgangsort & Zielort \\
\hline $\begin{array}{l}\text { apanhar } \\
\text { buscar } \\
\text { pegar }\end{array}$ & wo, woher & - \\
tomar & wo & wohin \\
levar & wo, woher & (wo) \\
trazer & woher & wo, wohin \\
woher & woher & wo, wohin \\
abholen & wo, woher & wo, wohin \\
bringen & wo, woher & wohin \\
mitbringen & woher & wohin \\
nehmen & woher & wohin \\
mitnehmen & woher & (wo), wohin \\
\hline
\end{tabular}

Situierung gibt, die typischerweise als erste gestellt wird. In unserem Korpus und in den Belegen der Wörterbücher kann man deutlich erkennen, dass die meisten und vor allem die als prototypisch angeführten Beispielsätze jeweils diese Fragen beantworten. In der Tabelle sind die betreffenden Frageadverbien durch Fettdruck hervorgehoben.

Zunächst fällt auf, dass im Portugiesischen bei allen sechs Verben wo-Bestimmungen möglich sind. In drei Fällen sind sie besonders typisch. Bei pegar kann (in verschiedenen Varianten des Verbs) der Ausgangsort oder der Zielort mit einer wo-Bestimmung spezifiziert werden:
a. Peguei ela na escola.
'Ich habe sie in der Schule abgeholt. $\rightarrow$ Ausgangsort'
b. Ele pegou cola na carta.
'Er hat Klebstoff auf den Brief geschmiert. $\rightarrow$ Zielort'

Im Deutschen sind demgegenüber nur bei vier der sechs Verben wo-Bestimmungen möglich, und wo ist in keinem Fall die typischste Frage. Bei mitbringen und mitnehmen sind weder in den Wörterbüchem noch in unserem Korpus wo-Bestimmungen belegt. Zumindest umgangssprachlich dürften sie aber möglich sein und sind auch eindeutig interpretierbar:

(18) Auf dieser Party muss jeder etwas zu essen mitbringen. (Zielort)

(19) Nimm auf dem Markt doch bitte ein paar Kilo Kartoffeln mit. (Ausgangsort) 
Im Portugiesischen gibt es zwei Verben, bei denen die wichtigste Frage die nach dem Zielort zu sein scheint, nämlich levar und trazer. Im Deutschen gibt es drei solche Verben, nämlich bringen, mitbringen und mitnehmen. Es ist zu vermuten, dass diese Verben in irgendeiner Weise Äquivalente bilden.

Unter den übrigen portugiesischen Verben ist nur eines, das sowohl woherals auch wohin-Bestimmungen zulässt, nämlich tomar. Eine ähnliche Spezifik besitzt im Deutschen nehmen. Bei den restlichen Verben (portugiesisch apanhar, buscar und pegar, deutsch holen und abholen) bestehen zwischen beiden Sprachen relativ große Unterschiede hinsichtlich der möglichen Ortsbestimmungen.

Wenn man das Korpus daraufhin untersucht, mit welcher Häufigkeit von den brasilianischen und deutschen Informanten in der Muttersprache wo-, woherund wohin-Bestimmungen zur Spezifizierung von Ausgangs- und Zielorten verwendet werden, so stellt man bei den brasilianischen Informanten eine Präferenz für Konstruktionen fest, in denen nur Subjekt- und Objekt-Komplemente vorkommen (ca. $60 \%$ ) gegenüber Konstruktionen, die außerdem wo-, woheroder wohin-Bestimmungen enthalten (ca. $40 \%$ ). Offenbar besteht im brasilianischen Portugiesisch eine Tendenz, Ausgangs- und Zielorte nicht über Komplemente von Transportverben zu spezifizieren, sondern im Kontext durch andere Mittel erkennbar zu machen. Wird ein Ausgangs- oder Zielort durch ein Verbkomplement spezifiziert, so wird in ca. $50 \%$ der Fälle eine wo-Bestimmung und in $50 \%$ eine woher- oder wohin-Bestimmung gewählt. Lediglich bei levar und (weniger deutlich) bei trazer findet man eine Präferenz für wohin-Bestimmungen.

Die deutschen Informanten zeigen in ihrer Muttersprache eine stärkere Tendenz, erstens Ausgangs- und Zielorte über wo-, woher- und wohin-Komplemente auszuweisen (in ca. $60 \%$ der Fälle gegenüber $40 \%$ reinen SubjektObjekt-Konstruktionen) und dies zweitens ganz überwiegend in Form von woher-oder wohin-Bestimmungen zu tun (wo-Bestimmungen in weniger als $5 \%$ ). Sogar bei Präfixverben, bei denen das Präfix bereits eine eindeutige woheroder wohin-Bestimmung liefert (hin/herholen, hin-/herbringen, wegnehmen, wegholen, wegbringen etc.) finden sich regelmäßig zusätzliche Wegbestimmungen:

Er brachte die Bücher wieder zur Bücherei hin.

In der Fremdsprache verwenden beide Informantengruppen relativ weniger wo-, woher- und wohin-Bestimmungen als in der Muttersprache. Wahrscheinlich führt Unsicherheit dazu, dass sie einfachere Strukturen bevorzugen. Deutsche Informanten geben dennoch beim Gebrauch der portugiesischen Verben immer noch mehr Ortsbestimmungen als Brasilianer. Dabei fallen vor allem zwei Besonderheiten auf: 
1. Deutsche Informanten verwenden bei portugiesischen Transportverben mehr woher- und wohin-Bestimmungen und weniger wo-Bestimmungen als Brasilianer, z. B. relativ häufiger apanhar de und relativ seltener apanhar $\mathrm{em}$. Dies ist eine Quelle für unidiomatische Ausdrucksweise.

2. Deutsche Informanten verwenden woher- und wohin-Bestimmungen auch bei Verben, die solche Bestimmungen nicht zulassen, wie z. B. woher-Bestimmungen bei buscar.

*Vou buscar o livro da minha casa.

Dies ist eine Quelle für Grammatikfehler.

Generell handelt es sich um eine Übertragung deutscher Sprachgebrauchskonventionen auf das brasilianische Portugiesisch (zielsprachexternes Motiv). Im Fall von apanhar und buscar liegt eine Interferenz mit den deutschen Verben holen bzw. abholen vor.

Brasilianische Informanten, die schon in ihrer Muttersprache weniger Ortsbestimmungen geben als deutsche Sprecher, geben noch weniger im Deutschen. $80 \%$ der von Brasilianern gebildeten Sätze mit deutschen Transportverben in unserem Korpus enthalten keine Ortsbestimmung. Von den übrigen $20 \%$ entfallen ca. drei Viertel auf woher- und wohin-Bestimmungen und ein Viertel auf wo-Bestimmungen. Obgleich der Anteil der wo-Bestimmungen damit deutlich niedriger liegt als in den von Brasilianern produzierten portugiesischen Sätzen, liegt er immer noch höher als in der Produktion der deutschen Informanten. Dafür liegt der Anteil der woher- und wohin-Bestimmungen bei weitem darunter.

Auch bei brasilianischen Informanten fallen somit zwei Besonderheiten auf: 1. Brasilianische Informanten verwenden bei deutschen Transportverben bei weitem weniger woher- und wohin-Bestimmungen und geringfügig mehr wo-Bestimmungen als deutsche. Dies ist eine Quelle für unidiomatische Ausdrucksweise.

2. Brasilianische Informanten verwenden häufig auch bei solchen Verben keine woher- bzw. wohin-Bestimmung, die eine solche Bestimmung fordern, wie z. B. bringen.

*Ich bringe die Hausaufgabe jede Stunde.

und wo-Bestimmungen bei Verben, die keine wo-Bestimmungen zulassen:

*Du bringst ein Hund zu Hause.

Dies ist eine Quelle für Grammatikfehler.

Auch hier handelt es sich um Übertragungen von Sprachgebrauchskonventionen aus der Muttersprache in die Fremdsprache (zielsprachexternes Motiv). Im Falle von bringen liegt eine Interferenz mit den portugiesischen Verben le- 
var und trazer vor, die beide sowohl mit einer wo-Bestimmung des Zielortes als auch ganz ohne Ortsbestimmung verwendet werden können.

Daneben kann der Verzicht auf woher- und wohin-Bestimmungen bei brasilianischen Informanten Ergebnis einer Fehlervermeidungsstrategie sein (zielsprachinternes Motiv: den komplizierten deutschen Präpositionen ausweichen).

Die dritte Beobachtung lautet mithin: Die Ortsbestimmungen sind bei den Transportverben eine typische Fehlerquelle sowohl für deutschlernende Brasilianer als auch für portugiesischlernende Deutsche.

\subsection{Wege und Perspektivierung}

Für die Selektion von Transportverben ist es wichtig zu wissen, was für Wege bei dem darzustellenden Sachverhalt von welchen Teilnehmern durchlaufen werden. Betrachten wir zum Beispiel die deutschen Verben holen und abholen im Vergleich zu bringen, mitbringen und mitnehmen. Die drei letzteren sind geeignete Darstellungsmittel, wenn ein Patiens $x$ einen Weg von A nach $B$ durchläuft, wobei als weiterer Sachverhaltsteilnehmer ein Agens auftritt, das den Gegenstand bewegt und das mit ihm zusammen denselben Weg durchläuft. So besagt etwa der Satz:

a. Der Ober bringt die Getränke an den Tisch.,

dass Ober und Getränke zusammen denselben Weg von einem nicht näher spezifizierten Ausgangsort an den Tisch (Zielort) beschreiben.

Dagegen kommen holen und abholen nur dann als Darstellungsmittel in Frage, wenn zunächst ein Weg vom Agens allein ohne Patiens zurïckgelegt, das Patiens am Ende dieses Weges vom Agens aufgenommen und von dort aus an einen dritten Ort bewegt wird. Im Falle von holen sollte dieser Zielort der Ausgangsort des Agens sein, d.h. das Agens durchläuft zweimal den gleichen Weg in verschiedenen Richtungen. So besagt etwa der Satz:

b. Peter holt die Getränke an der Theke.,

dass Peter aufsteht, den Weg zur Theke zurücklegt und von dort aus zusammen mit den Getränken wieder seinen Ausgangsort, zum Beispiel seinen Tisch, aufsucht.

Im Falle von abholen ist diese Variante möglich, aber nicht notwendig, d.h. das Agens kann zwei verschiedene Wege durchlaufen und den Gegenstand an einen Zielort bewegen, der nicht sein eigener Ausgangsort ist. So kann der Satz:

c. Peter holt die Getränke an der Theke ab. 
zum Beispiel auch verwendet werden, wenn Peter von einem Tisch, an dem er gewartet hat, aufsteht, an die Theke geht, dort die vorher bestellten Getränke in Empfang nimmt und sich mit ihnen hinaus auf die Straße begibt.

Im Portugiesischen finden wir eine ähnliche Verteilung bei buscar und apanhar. Buscar ist als Darstellungsmittel geeigneter, wenn der gleiche Weg zweimal durchlaufen wird (wie bei holen); apanhar lässt offen, ob der AgensTeilnehmer an seinen Ausgangsort zurückkehrt oder nicht (wie abholen). Somit sollten buscar und apanhar, die unter dem Gesichtspunkt der Konstruktion (wo-, woher- und wohin-Bestimmungen) für portugiesischlernende Deutsche Fehlerquellen bilden, unter dem Gesichtspunkt der Selektion weitgehend unproblematisch sein. Dies wird durch unsere Daten in der Tat bestätigt.

Anders liegen die Verhältnisse bei den Darstellungsmitteln für Transportsachverhalte, bei denen Agens und Gegenstand zusammen nur einen Weg durchlaufen. Im Portugiesischen stehen hier die Verben levar und trazer zur Verfügung, im Deutschen, wie schon erwähnt, die Verben bringen, mitbringen und mitnehmen.

Für die Selektion von levar und trazer im Portugiesischen ist in erster Linie die Situierung des Sprechers relevant, d.h. diese Verben werden gewöhnlich deiktisch verwendet. Ist der Sprecher am Ausgangsort des Weges situiert, den der Transport-Gegenstand durchläuft, so muss levar verwendet werden. Trazer ist in solchen Fällen inakzeptabel. Ist der Sprecher dagegen am Zielort des Weges situiert, muss trazer verwendet werden. Dann ist levar inakzeptabel.

Der folgende Dialog zwischen zwei Muttersprachlerinnen des brasilianischen Portugiesisch (Originalbeleg der Projekt-Mitarbeiterin Glauce Rocha de Oliveira) veranschaulicht besonders deutlich die unterschiedliche Verwendungsweise der beiden Verben:

$$
\begin{aligned}
& \mathrm{S} 1 \text { - Paloma, você pode trazer o dicionário? 'Paloma, kannst du } \\
& \text { bitte das Wörterbuch mit herbringen?' } \\
& \mathrm{S} 2 \text { - } \quad \text { Ah, trago - eh, levo. 'Ja, ich bringe es her - eh, mit.' }
\end{aligned}
$$

Es handelt sich um einen Ausschnitt aus einem Telefondialog, in dem die Sprecherin S2 zunächst das von S1 vorgegebene Verb trazer wiederholt, dann aber erkennt, dass dieses in der Antwort die falsche Perspektive induziert und es daher durch levar korrigiert.

Wenn der Sprecher sich weder am Ausgangs- noch am Zielort befindet, ist die Entscheidung zwischen levar und trazer eine Frage der Perspektivierung des Weges. In diesem Fall orientiert levar den Rezipienten in Wegrichtung (Betrachtung des Weges vom Ausgangsort aus) und trazer in Gegenrichtung (Betrachtung vom Zielort aus). Ein illustratives Beispiel für diesen Gebrauch der Verben gibt das Dicionário Gramatical de Verbos do Português Contemporâneo do Brasil (Borba et al. 1991: 1317): 
Qualquer doença que traga vômito ou diarréia leva à desidratação. 'Jede Krankheit, die Erbrechen oder Durchfall mit sich bringt, führt zu Flüssigkeitsverlust.'

Hier wird als Orientierungspunkt der Kranke gewählt, also das Ziel von Erbrechen und Durchfall (Perspektive entgegen der Wegrichtung) und derjenige, der zum Flüssigkeitsverlust geführt wird (Perspektive in Wegrichtung).

Dass Sprechersituierung und Wegperspektivierung als Kriterien für die Selektion von levar und trazer in Konflikt geraten können, zeigt die Selbstkorrektur in Beispiel (25). Nach der schriftsprachlichen Norm erlauben levar und trazer keine Verwendung mit adressatenzentriertem Bezugsrahmen (zur Terminologie vgl. Blühdorn 1993b: 56 ff.), also keine Übernahme der Adressatenperspektive. Im gesprochenen brasilianischen Gegenwartsportugiesisch scheinen bezüglich dieser Beschränkung aber gewisse Unsicherheiten zu bestehen. Selbstkorrekturen wie (25) sind bei Muttersprachlern keine Seltenheit.

Für den Gebrauch von Ortsbestimmungen ist bei levar, das den Weg vom Ausgangsort aus perspektiviert, zu erwarten, dass es häufiger als andere Transportverben durch wohin-Bestimmungen ergänzt wird. Diese Erwartung wird durch die Wörterbücher wie auch durch die Produktion unserer brasilianischen Informanten in vollem Umfang bestätigt. Für trazer, das den Weg vom Zielort aus perspektiviert, wären entsprechend relativ häufiger woher-Bestimmungen zu erwarten. Dies wird teilweise durch die in den Wörterbiichern gegebenen Beispiele, nicht aber durch unsere brasilianischen Informanten bestätigt. In unserem Korpus steht bei trazer bei weitem der Gebrauch ohne Wegbestimmung (zwei Drittel aller Beispiele), ansonsten die Ergänzung von wohin-Bestimmungen im Vordergrund. Um diesen Befund zu interpretieren, ist es vielleicht hilfreich sich klarzumachen, dass wohin-Bestimmungen bezüglich der Richtung unserer alltäglichen Handlungsorientierung und Ereigniserfahrung ikonisch sind, während woher-Bestimmungen sich in dieser Hinsicht anti-ikonisch verhalten. Nicht nur im Portugiesischen, sondern auch im Deutschen und in anderen Sprachen scheinen wohin-Bestimmungen generell häufiger vorzukommen als woher-Bestimmungen (vgl. auch den in der Linguistik üblichen Terminus "Direktionalbestimmung" für "Wegbestimmung"). Unter der zeichentheoretisch plausiblen Annahme, dass es in natïrlichen Sprachen etwas wie eine generelle Ikonizitäts-Tendenz gibt, erscheint die Asymmetrie zwischen levar und trazer in diesem Punkt zumindest verständlicher.

Die wichtigsten deutschen Äquivalente für levar sind mitnehmen und bringen. Für beide ist die Perspektivierung in Bewegungsrichtung kennzeichnend. Mitnehmen ist typischer wenn der Ausgangsort der Bewegung zugleich der Sprecherort ist, aber es kann auch verwendet werden, wenn Ausgangsort und Sprecherort verschieden sind. Bringen ist umgekehrt typischer, wenn Ausgangsort und Sprecherort verschieden sind, aber es ist auch möglich, wenn bei- 
de zusammenfallen. Ein wichtiger Unterschied zwischen beiden Verben liegt in der relativen Gewichtung von Agens-Bewegung und Gegenstands-Bewegung. Bringen wird gewählt, wenn die Agens-Bewegung nur um der Gegenstands-Bewegung willen ausgeführt wird; mitnehmen wird gewählt, wenn die Agens-Bewegung auch sonst erfolgt wäre:

a. Die Mutter hat die Kinder in die Schule gebracht.

b. Die Mutter hat die Kinder in die Schule mitgenommen.

In (27a) versteht man, dass die Mutter sich eigens auf den Weg gemacht hat, um die Kinder zur Schule zu befördern, während man in (27b) versteht, dass sie sowieso gefahren wäre und die Gelegenheit für den Transport der Kinder ausgenutzt hat. Bei bringen und mitnehmen spielen somit konzeptuelle Beschränkungen eine Rolle, die im Portugiesischen irrelevant sind.

Das wichtigste Äquivalent für trazer ist mitbringen. Beide Verben perspektivieren den Weg vom Zielort aus, wobei der Zielort der Sprecherort oder ein im Textzusammenhang gegebener Orientierungsort sein kann. Bei mitbringen kommt ferner, anders als bei trazer nach der portugiesischen Schriftsprachnorm, auch der nicht mit dem Sprecherort übereinstimmende Adressatenort als Zielort in Frage. Deshalb wäre in einem Fall wie (25) im Deutschen mitbringen in beiden Redebeiträgen voll akzeptabel. Mitbringen ist hinsichtlich des deiktischen Bezugsrahmens flexibler als trazer.

Levar und trazer, unter dem Gesichtspunkt der Konstruktion weitgehend unproblematisch, bereiten deutschen Portugiesischlernern Schwierigkeiten unter dem Gesichtspunkt der Selektion. Typische Fehler aus unserem Korpus sind:

Espera um pouco, vou levar um bocadinho de água.

'Warte mal, ich hole ein bisschen Wasser.' statt: buscar

Você vai trazer o livro a sua viagem?

'Nimmst du das Buch mit auf die Reise?' statt: levar

In erster Linie sind die Schwierigkeiten wohl darauf zurückzuführen, dass im Portugiesischen zunächst der Sprecherort und dann der Orientierungsort die Selektion bestimmt, während im Deutschen stets der Orientierungsort maßgeblich ist. Bei trazer kommt die Beschränkung hinsichtlich des adressatenzentrierten Bezugsrahmens hinzu. Darüber hinaus wird die Selektion sicher auch durch die asymmetrischen Äquivalenzbeziehungen zwischen den portugiesischen und deutschen Verben erschwert. Zum einen entsprechen den portugiesischen Simplizia levar und trazer zwei deutsche Präfixverben mitnehmen und mitbringen. Zum anderen entspricht das Simplex des zweiten (bringen) dem portugiesischen Äquivalent des ersten (levar), während das andere deutsche Simplex (nehmen) ganz aus der Äquivalenz herausfällt. 
Brasilianische Deutschlerner haben mit der Wegperspektivierung im Deutschen demgegenüber keine Schwierigkeiten. Da das Deutsche mehr Möglichkeiten bietet als das Portugiesische, lassen sie allenfalls einige davon ungenutzt. Für sie verbindet sich die Asymmetrie im lexikalischen Bereich mit der Schwierigkeit, deutsche Präfixverben zu benutzen (zu den Perspektivierungseigenschaften der Verbpräfixe vgl. Eichinger 1989). Sie verwenden sowohl für levar als auch für trazer bevorzugt bringen - im Falle von trazer Simplex des Äquivalentes mitbringen, im Falle von levar legitimes Äquivalent, das gegenüber mitnehmen um so leichter bevorzugt werden kann, als die relative Gewichtung von Agens-Bewegung und Gegenstands-Bewegung als Selektionskriterium im Portugiesischen unbekannt ist.

Die vierte Beobachtung lautet mithin: Der deiktische Gebrauch der Transportverben ist eine typische Fehlerquelle für portugiesischlernende Deutsche. Maßgeblich dafür ist die geringere Flexibilität der portugiesischen Verben hinsichtlich des deiktischen Bezugsrahmens (zielsprachexternes Motiv). Deutschlernende Brasilianer scheitern nicht an der im Deutschen flexibleren Deixis, sondern an den komplizierten Äquivalenzbeziehungen zwischen deutschen Präfixverben und Simplizia und portugiesischen Simplizia (ebenfalls ein zielsprachexternes Motiv).

\section{Resümee}

Wir haben gesehen, dass System und Verwendung der Transportverben im Deutschen und im brasilianischen Portugiesisch sich unter grammatischem, konzeptuell-semantischem und pragmatischem Blickwinkel unterscheiden und eine Fülle von Fehlerquellen für Fremdsprachenlerner in beiden Richtungen bereithalten. Fehler können zum einen manifeste Verstöße gegen Regeln sein; es kann sich aber auch um unidiomatischen Gebrauch handeln, der zum Beispiel durch höhere oder niedrigere Frequenz bestimmter Arten von Ortsbestimmungen auffällt.

Die in der vorliegenden Arbeit präsentierten vier Beobachtungen deuten darauf hin, dass die Hauptprobleme für Brasilianer mit den deutschen Transportverben in der Grammatik liegen (Präfixe, Präpositionen, Deklination), die Hauptprobleme für Deutsche mit den portugiesischen Transportverben dagegen in der Pragmatik (Wege und Perspektivierung). Im Bereich der Semantik (Bestimmung von Situierungs-, Ausgangs- und Zielorten) gibt es Probleme in beiden Richtungen, die aber überwiegend zu unidiomatischem und nur vereinzelt zu manifest falschem Gebrauch führen.

\section{Anmerkungen}

1. An diesem Projekt wirkten Glauce Rocha de Oliveira, Luis Fernando Dias Moreira und Renato Ferreira da Silva als wissenschaftliche Hilfskräfte mit. Ihre Tätig- 
keit wurde durch die Fundação de Amparo à Pesquisa do Estado de São Paulo [Forschungsförderungsstiftung des Bundeslandes São Paulo] (FAPESP) finanziert (Vorg.-Nrn. 96/03305-0 und 96/03306-6).

2. Ich danke Bernd Naumann und Carlos Schünemann von der Universität ErlangenNürnberg, Günter Kipfmüller und den Lehrkräften des Goethe-Institutes São Paulo sowie meinen KollegInnen vom Institut für Deutsch der Universität São Paulo für ihre Hilfe bei der Erhebung der Daten.

Neben dem schon erwähnten Material enthält das Korpus einen zweiten Teil von nochmals ca. 2.500 Sätzen, der aus Beschreibungen von Bildgeschichten durch Muttersprachler und Fremdsprachler in deutscher und portugiesischer Sprache besteht. Diese Daten wurden für die im vorliegenden Aufsatz diskutierten Punkte aber nicht ausgewertet.

3. Ich zähle hier die sogenannten Partikelverben (vgl. Duden 1998: 44) einfachheitshalber zu den Präfixbildungen.

4. Em wird an den Definitartikel $o$ bzw. $a$ klitisiert. Das gleiche geschieht mit den Präpositionen de und $a$ sowie mit de bei dem Adverb aqui [hier].

5. Eine große brasilianische Internet-Suchmaschine trägt den Namen Aonde.

6. Beispiel (17b), das aus einem Wörterbuch stammt, wurde allerdings von unseren brasilianischen Informanten durchweg abgelehnt. Es dürfte sich um europäisches Portugiesisch handeln.

\section{Literaturverzeichnis}

Blühdorn, Hardarik (1993a). Funktionale Zeichentheorie und deskriptive Linguistik. Ein Entwurf am Beispiel des Gegenwartsdeutschen. Erlangen, Palm \& Enke.

- (1993b). "Deixis und Deiktika in der deutschen Gegenwartssprache". In: Deutsche Sprache 21: 44-62.

Borba, Francisco da Silva et al. (1991). Dicionário Gramatical de Verbos do Português Contemporâneo do Brasil. 2a. ed., São Paulo, UNESP.

Buarque de Holanda Ferreira, Aurélio (1986). Novo Dicionário da Língua Portuguesa. 2a. ed., Rio de Janeiro, Nova Fronteira.

Duden (1989). Deutsches Universalwörterbuch. 2. Aufl., Mannheim, Bibliographisches Institut.

Duden (1998). Grammatik der deutschen Gegenwartssprache. 6. Aufl, Mannheim, Bibliographisches Institut.

Eichinger, Ludwig M. (1989). Raum und Zeit im Verbwortschatz des Deut-schen. Eine valenzgrammatische Studie. Tübingen, Niemeyer.

Engel, Ulrich and Helmut Schumacher (1978). Kleines Valenzlexikon deutscher Verben. Tübingen, Narr.

Fernandes, Francisco (1991). Dicionário de verbos e regimes. 38a. ed., São Paulo, Globo.

Fleischer, Wolfgang and Irmhild Barz (1992). Wortbildung der deutschen Gegenwartssprache. Tübingen, Niemeyer.

Gerling, Martin and Norbert Orthen (1979). Deutsche Zustands- und Bewegungsverben. Eine Untersuchung zu ihrer semantischen Struktur und Valenz. Tübingen, Narr.

Götz, Dieter et al. (Hg.) (1993). Langenscheidts Großwörterbuch Deutsch als Fremdsprache. Das neue einsprachige Wörterbuch für Deutschlernende. Berlin, Langenscheidt.

Helbig, Gerhard and Joachim Buscha (1986). Deutsche Grammatik. Ein Handbuch für den Ausländerunterricht. 9. Aufl., Leipzig, VEB Verlag Enzyklopädie.

Helbig, Gerhard and Wolfgang Schenkel (1973). Wörterbuch zur Valenz und Distribution deutscher Verben. 2. Aufl., Leipzig, VEB Bibliographisches Institut. 
Irmen, Friedrich and Albin Eduard Beau (1986). Langenscheidts Taschenwörterbuch Portugiesisch-Deutsch/Deutsch-Portugiesisch. 4./17. Aufl., Berlin Langenscheidt.

Keller, Alfred J. (1994). Michaelis. Pequeno Dicionário Alemão-Português/Português-Alemão. São Paulo, Melhoramentos.

Klare, Johannes et al. (1988). Wörterbuch Portugiesisch-Deutsch. 2. Aufl,, Leipzig, VEB Verlag Enzyklopädie.

Luft, Celso Pedro (1995). Dicionário Prático de Regência Verbal. 3a. ed., São Paulo, Ática.

Schau, Udo (1985). Dicionário de Alemão-Português. Porto, Editora Porto.

Schröder, Jochen (1992). Lexikon deutscher Präfuxverben. Berlin, Langenscheidt Enzyklopädie.

Schumacher, Helmut (Hg.) (1986). Verben in Feldern. Valenzwörterbuch zur Syntax und Semantik deutscher Verben. Berlin, de Gruyter. 